\title{
Irreversibility in river flow
}

\author{
Umberto Lucia ${ }^{a}$, Patrizia Buzzi ${ }^{b}$, Giuseppe Grazzini ${ }^{c}$ \\ ${ }^{a}$ Dipartimento Energia, Politecnico di Torino, Corso Duca degli Abruzzi24, 10129 Torino, Italy, umberto.lucia@polito.it \\ ${ }^{b}$ Regione Piemonte, Opere Pubbliche e Difesa Assetto Idrogeologico, Ufficio di Alessandria, Piazza Turati 5, 15121 \\ Alessandria, Italy ${ }^{c}$ Dipartimento di Ingegneria Industriale, Via S. Marta 3, 50139 Firenze, Italy
}

\begin{abstract}
In the last twenty years, the entropy generation approach has been developed in order to analyse complex systems (open and irreversible). The first applications of the entropy generation extrema theorem were developed on energy systems, but recently, it has also been extended to biological systems in order to evaluate their stationary states. In this paper, the entropy generation is analyzed in relation to the hydrological and hydraulic behaviour of rivers. An example is developed on the Tanaro river in Piedmont (Italy).
\end{abstract}

Keywords: Entropy, environment, flood, irreversibility, river.

\section{Introduction}

A sustainable approach to water management [1] is a fundamental topic in the contemporary society. The concept of Integrated Water Resources Management was introduced in order to incorporate the multiple competing uses of water resources at the United Nations Conference on Water in the Mar del Plata (1977). In the 1990s, at the "International Conference on Water and Environment" (1992), at the "Second World Water Forum" (2000), in the "International Conference on Freshwater" (2001), at the "World Summit on Sustainable Development" (2002) and at the "Third World Water Forum" (2003), its fundamental role in sustainability was pointed out [2].

In history there have always been flood events, and today they continue to be, as a consequences of natural behaviour of rivers and water flows during particular atmospheric events. Consequently, when there exists human establishment flood prevention or mitigation plans must be developed in order to promote and manage 
actions regarding water, land and related resources. But these actions should cover the entire river basin, by using a global approach which allows us to consider it as a complex system, where different phenomena occur: flooding, erosion, mass deposition, landslides, etc.. It has been pointed out that mitigation measures tend to be potentially more efficient and long term more sustainable solutions to flood problems and should be developed in order to reduce the vulnerability of human beings and goods exposed to flood risk. Indeed, even if defence structures are important elements of the protection of human safety, it has been underlined that flood protections are never absolute, and defence structures can generate a false sense of security in the people. The concept of residual risk must always be considered. So, flood forecasting and warning are fundamental in order to obtain successful mitigation of flood damage [3]. In this context, the quantitative evaluation of discharge at river sites is fundamental in order to develop the water resource management both for its use and to prevent the floods. But, the discharge evaluation is related to the velocity measurements, often restricted by the excessive costs involved. Consequently, many mathematical physical models have been developed in order to evaluate the velocity profiles distribution, but only few studies obtained the spatial velocity distribution during high flood conditions [4]. But, the useful information on discharge is the water flow. So, instead to evaluate the velocity it could be useful an approach to the river hydraulic behaviour to obtain just the water flow.

Last, during floods, it is fundamental to be able to evaluate the area interested by the phenomena and the time in which they will occur, in order to work for people safety and damages prevention.

Nature is governed by the second law of thermodynamics, with particular regards to irreversibility. Entropy has been successfully introduced in the hydraulic and hydrological analyses [4-6] pointing out how this quantity can be effective in these studies. In the last two decades many results have been obtained just in irreversible thermodynamics useful to evaluate quantitatively many natural phenomena [7-20].

The aim of this paper is to present the entropy generation extrema theorem [15] use in river flows analysis as a first step for a future possible quantitative approach to hydrology and hydraulics in order to evaluate the evolution and the behaviour of water flows [8] and effects. To do so in Section 2 the entropy generation approach is developed, in Section 3 it is used to analyze the behaviour of Tanaro river.

\section{Entropy generation approach}

Earth system is far from equilibrium as pointed out by Lovelock [21]. The hydrologic cycle is caused by the vapour concentration out of equilibrium, driven by [6]:

$\circ$ Updrafts: the vapour is brought to supersaturation

- Precipitation: the vapour becomes unsaturated when it descends back to the Earth surface

- Potential energy on the Earth surface associated with the different height

- Different water state of matter due to temperature gradient on the Earth surface. 
All these disequilibria represent the source of exergy flows due to energy gradients [22-27] associated with them [17].

In order to study the dissipation of fluid systems entropy has been introduce [28$30]$, for any volume $V$ considered:

$\int_{V} \dot{s} d V=\int_{V} \frac{\dot{q}}{T} d V+\int_{V} \frac{\dot{w}_{\lambda}}{T} d V$

where $\dot{s}$ is the specific entropy, $V$ is the volume, $\dot{q}$ is the specific heat power absorbed, $T$ is the temperature and $\dot{w}_{\lambda}$ is the specific lost mechanical power, and such that it is always verified that:

$\int_{V} \dot{s} d V>\int_{A} \frac{J_{\dot{Q}}}{T} d A$

with $J_{\dot{Q}}$ heat power flux density.

Gibbs introduced the available energy, named exergy, defined as the maximum useful work obtained if a system is in thermodynamic equilibrium with the environment with a process in which the system interacts only with it [29]. The available work lost in an irreversible process, the exergy lost, called also as anergy or exergy dissipation, can be obtained as:

$$
E_{\lambda}=\int_{0}^{\tau}\left(\dot{E}_{W}^{r e v}-\dot{E}_{W}\right) d t=W_{\lambda}=T_{0} S_{g}
$$

This relation is known as the Gouy-Stodola theorem [31-33]. It represents a fundamental approach in engineering analysis of the irreversibility of open systems, useful to evaluate the work lost for irreversibility using the entropy generation balance [17].

Starting from this theorem, since 1995 we are carrying on a theoretical and phenomenological approach based on entropy generation in order both to introduce a principle of analysis for irreversibility in engineering and science [15] and to obtain a computational approach related to the 'natural' behavior of the phenomena. The mathematical basis of this approach is the variational calculus, while the physical basis is the principle of least action and the Noether's theorem too, and some phenomenological hypothesis as follows [15]:

1. a real open irreversible linear or non-linear system is considered;

2. each process has a finite lifetime $\tau$,

3. what happens in each instant in the range $[0, \tau]$ cannot be known, but what was happened after the time $\tau$ (the result of the process) is well known (at least it is sufficient to wait and observe is required only to define temperature and entropy;

4. the entropy balance equation is a balance of fluxes of entropy and energy. The result obtained consists in the conditions of stability of a stationary state for an open system [15]:

$S_{g} \leq T_{0} \frac{T_{1}-T_{0}}{T-T_{0}} W_{\lambda 1} \quad \forall T \neq T_{0}$

where $\mathrm{W}_{\lambda 1}$ is the lost work required to maintain the state 1 of the open system at the temperature $T_{1} \neq T_{0}$, with $T_{0}$ environment temperature. This relation proves that: 
○ the entropy generation has an upper limit for non equilibrium states;

$\circ$ the entropy generation rate has a maximum for the stationary states (sign $=)$.

The relation (8) underlines that the entropy generation $S_{g}$ due to the transition to a new state at temperature $\mathrm{T}$ is related to the initial state conditions. Therefore, it represents a selection rule in relation to the initial and final states: not all the thermodynamic path are allowed and the most probable path [16] is the one that satisfies the relation (8) with the sign " $="$. Now, considering that, in the stationary states the entropy generation is at an extremum [7,10], it can be derived the following relations:

$$
\delta S_{g}=\frac{\partial S_{g}}{\partial t} d t+\nabla_{\zeta} S_{g} \cdot d \zeta=0 \Rightarrow \frac{\partial S_{g}}{\partial t}=-\nabla_{\zeta} S_{g} \cdot \dot{\zeta}
$$

where $\zeta$ is the considered spatial variables ( $x$ or $V$, etc.) and:

on least path $\Rightarrow S_{g}=\max _{\tau} \Rightarrow \frac{\partial S_{g}}{\partial t} \geq 0$

then it follows:

$$
\left.\left.\begin{array}{l}
\frac{\partial S_{g}}{\partial t}=-\nabla_{\zeta} S_{g} \cdot \dot{\zeta} \\
\frac{\partial S_{g}}{\partial t} \geq 0
\end{array}\right\} \Rightarrow \begin{array}{c}
-\nabla_{\zeta} S_{g} \cdot \dot{\zeta} \geq 0 \\
\dot{\zeta}>0
\end{array}\right\} \Rightarrow \nabla_{\zeta} S_{g} \leq 0
$$

from which it is possible to argue if the open system evolves also in space to attain the stationary state, the entropy generation results maximum or minimum in consequence of the prevailing of the spatial or time term.

In order to use this principle in relation to hydraulics and hydrology, it is possible to state that the hydrological or hydraulic system must satisfy the relations (4) and (7) and in particular that the system is stationary if

$\nabla S_{g} \leq 0$

The entropy generation approach is very interesting because it:

○ introduces the time-life of the process, the time of occurrence of a process;

- analyzes the systems in a time greater than or equal to the lifetime of the entire process occurred in the system.

\section{Entropy generation in hydraulics and hydrology}

The entropy generation approach can allow us to obtain information on the stationary states of rivers, channels, hydro-morphological states of systems, etc. As example this principle will be used to evaluate the stability of the Tanaro river in Piedmont (Italy). 
First, for a river, the hydraulic entropy $S_{h}$ is defined as [28]:

$\frac{d S_{h} / d t}{\dot{V}}=\frac{d H / d x}{H}$

with t time, $\dot{V}$ river discharge (volume flow), $\mathrm{H}$ elevation above the base level.

But, the entropy generation $\mathrm{S}_{\mathrm{g}}$ can be written as follows [18-20]:

$S_{g}=\frac{W_{\lambda}}{T}=\int_{0}^{\tau} \frac{\rho g}{T} \dot{\mathbf{V}} \cdot \nabla_{\dot{\mathbf{v}}} H d t$

where $\nabla_{\dot{v}}$ is the gradient evaluated in the direction of the flow; in a onedimensional simplified model it results:

$S_{g}=\int_{0}^{\tau} \frac{\rho g}{T} \dot{V} \frac{d H}{d x} d t$

Now this relation will be considered on the Tanaro river. It is a $276 \mathrm{~km}$-long river in northwestern Italy. It rises at the confluence of two small torrents whose sources are in Piedmont, the Tanarello and the Nerone, in the Ligurian Alps, close to the border with France and is the most significant right-side tributary to the Po in terms of length, size of drainage basin and discharge. The river is highly prone to flooding. During the two hundred year period 1801-2001 parts of the Tanaro basin were affected by floods on 136 occasions. The most recent and devastating occurred on November 1994 when the whole of the river valley was affected by severe flooding and the town of Alessandria was especially stricken. Consequently, it is important to obtain a model useful to evaluate the behaviour of Tanaro river, in order to prevent possible calamities.

The data considered are the ones collected by the two hydrometers in Alba (172 m on the sea level) and Masio (142 $\mathrm{m}$ on the sea level). The distance between the two city is around $34.4 \mathrm{~km}$. In stationary state, we expect that the entropy generation of the Tanaro river between the two cities is constant in time. They are represented in Figure 1. Following the previous results, it is possible to state that, during a calamity the expectation is that the entropy generation rate between the two places becomes negative and then change its sign to becomes positive and then constant again at the end of the calamity. Greater the difference more intense the calamity.

We have evaluated the entropy generation for Tanaro using the data collected and reported by ARPA-Piemonte [34]. The analysis obtained confirms the approach suggested. Indeed, Figure 2 represents the entropy generation evaluated in Masio and Alba, while the Figure 3 the variation of entropy generation in Masio and Alba. The entropy generation reach a maximum value during the maximum value of flood, represented in Figure 4, that underlines the sign of the entropy variation that gives information on the behaviour (increasing or decreasing of the flood). In Figure 5 the entropy variation (its gradient) on the path between Masio and Alba is represented. As expected it is negative during the increasing of the flood, null at the maximum flood and positive when the flood decreases. 


\section{Conclusions}

Any effect in Nature is the consequence of the interplay and dynamic balance between pairs of opposite elements of the interaction between the systems and the environment [35-37]]. Annila [22] pointed out that the natural behavior of the open systems is ascribed to the decrease of free energy in the least time, which can be calculated by the extrema entropy generation theorem here summarized [15]: this principle is interesting in science and engineering because it allows us to obtain a range in which an open system persists in a stationary state.

In this paper this approach is introduced in order to evaluate the behavior of hydrologic and hydraulic systems for the forecasting and prevention of any eventual disaster caused by water flows and flood. The analysis of the Tanaro flood between two hydrometric measurement stations is developed as example of use of this approach. The results on the data collected during a disaster agree with the evaluation obtained by the entropy generation approach, proving its use. Indeed, the entropy generation for Tanaro was evaluated by using the data collected and reported by ARPA-Piemonte [34]. The results obtained are represented in Figure 2-5 and can be summarized as follows:

- Figure 2 shows the entropy generation evaluation in Masio and Alba. It is the behaviour of the flow in the two villages and it reach a maximum at the stationary state, corresponding to the stationary flow, but this step is fundamental only to evaluate the variation of the entropy generation, the basic quantity used in the approach;

- In the Figure 3, the variation of the entropy generation in Masio and Alba is represented. The entropy generation reaches a maximum value during the maximum value of flood. This information is important because underlines how the analysis of the entropy generation shape for a river is fundamental in order to evaluate the behaviour of the flows;

- In Figure 4, the sign of the entropy generation variation rate is represented. The study of the sign is the fundamental step of the water flow analysis because it allows to obtain information on the increasing or decreasing of the flood;

In Figure 5, the entropy generation gradient on the path between Masio and Alba is represented. It is negative during the increasing of the flood, null at the maximum flood and positive when the flood decreases, suggesting how to forecast to the behaviour of the water flow.

- Last, the analysis of the entropy generation related to the rivers can be useful to simulate the flood behaviour and to underline the more sensitive area where engineers can work to prevent or reduce disasters.

\section{References}

[1] Diaz-Delgado, C., Antón, D., Esteller, M.V., García, J.A., Bâ, K.M., Quentin, E., Entropic Management of Water in Decentralised Countries. International Conference on Water Management in Federal and Federal-Type Countries, 
Zaragoza, Spain, 2008,

http://www.forumfed.org/en/global/thematic/water_papers/Carlos\%20Diaz\%20en final.pdf

[2] Rahaman, M.M., Varis, O., Integrated water resources management: evolution, prospects and future challenges, Sustainability: Science, Practice, \& Policy 1(1), 15-21, 2005.

[3] Yen, B.C., Hydraulics and effectiveness of levees for flood control, U.S.-Italy Research Workshop on the Hydrometeorology, Impacts, and Management of Extreme Floods, Perugia (Italy), November 1995, 1-12.

[4] Moramarco, T., Ammari, A., Burnelli, A., Mirauda, D., Pascale, V., Entropy Theory Application for Flow Monitoring in Natural Channels, IEMSs 2008: International Congress on Environmental Modelling and Software Integrating Sciences and Information Technology for Environmental Assessment and Decision Making. 4th Biennal Meeting of IEMSs. Sànchez-Marré, M., Béjar, J., Comas, J., Rizzoli, A., \& Guariso, G. Eds. International Environmental Medelling and Software Society, 2008, 430-437.

[5] Leopold, L.B., Langbein, W.B., The Concept of Entropy in Landscape Evolution. In Theoretical Papers in the Hydrology and Geomorphic Sciences. Geological Survey Professional Paper 500-A. United State Government Printing Office, Washington, 1962.

[6] Kleidon, A., Non-equilibrium thermodynamics, maximum entropy production and Earth-system evolution, Philosophical Transaction of the Royal Society A 368, 181-196, 2010.

[7] Lucia, U., Mathematical consequences and Gyarmati's principle in Rational Thermodynamics, Il Nuovo Cimento B 110(10), 1227-1235, 1995.

[8] Lucia, U., Maximum principle and open systems including two-phase flows, Revue Gènèrale de Thermique (today International Journal of Thermal Sciences), 37, 813-817, 1998.

[9] Lucia, U., Irreversibility entropy variation and the problem of the trend to equilibrium, Physica A 376, 289-292, 2007.

[10] Lucia, U., Probability, ergodicity, irreversibility and dynamical systems, Proceedings of the Royal Society A 464, 1089-1184, 2008 a.

[11] Lucia, U., Statistical approach of the irreversible entropy variation, Physica A 387(14), 3454-3460, 2008b.

[12] Lucia, U., Irreversibility, entropy and incomplete information, Physica $A$ 388, 4025-4033, 2009.

[13] Lucia, U., Maximum or minimum entropy generation for open systems?, Physica A 391(12), 3392-3398, 2012a.

[14] Lucia, U., Irreversibility in biophysical and biochemical engineering, Physica A 391, 5997-6007, 2012b.

[15] Lucia, U., Stationary open systems: a brief review on contemporary theories on irreversibility, Physica A 392(5), 1051-1062, $2013 a$. 
[16] Lucia, U., Irreversible human brain, Medical Hypothesis 80(2), 114-116, $2013 b$.

[17] Lucia, U., Entropy and exergy in irreversible renewable energy systems, Renewable \& Sustainable Energy Reviews 20, 559-564, $2013 \mathrm{~d}$.

[18] Lucia, U, Thermodynamic paths and stochastic order in open systems, Physica A 392, 3912-3919, 2013e.

[19] Lucia, U., Sciubba, E., From Lotka to the entropy generation approach, Physica A 392, 3634-3639, 2013.

[20] Lucia, U., Buzzi, P., La generazione di entropia nella analisi del comportamento delle acque fluviali. In Atti del Convegno L'alluvione del 5-6 novembre 1994 in Piemonte. Venti anni di attività nella previsione e prevenzione in ambito geo-idrologico. Torino 28-29 ottobre 2014. GEAM, Associazione Georisorse e ambiente, 167-172.

[21] Loveloch, J.E., A physical basis for life detection experiments, Nature 207, 568-570, 1965.

[22] Annila, A., Kuismanen, E.Natural hierarchy emerges from energy dispersal. BioSystems 95, 227-233, 2009.

[23] Kaila, V.R.I., Annila, A., Natural selection for least action, Proc. R. Soc. A. 464, 3055-3070, 2008.

[24] Hartonen, T., Annila, A., Natural networks as thermodynamic systems, Complexity 18, 53-62, 2012.

[25] Mäkelä, T., Annila, A., Natural patterns of energy dispersal, Phys. Life Rev. 7, 477-498, 2010.

[27] Würtz, P., Annila, A., Ecological succession as an energy dispersal process, BioSystems 100, 70-78, 2010.

[28] Marchi, E., Rubatta, A., Meccanica dei fluidi. Principi e applicazioni, Torino, Utet, 1981.

[26] Sharma, V., Annila, A., Natural process - Natural selection, Biophys. Chem. $127,123-128,2007$.

[29] Sciubba, E., Wall, G., A brief Commented History of Exergy From the Beginnnings to 2004, Int. J. Thermodynamics 10(1), 1-26, 2007.

[30] Bejan, A., Advance Engineering Thermodynamics, 3rd Ed., John Wiley, 2006.

[31] Gouy, G., Sur les transformation et l'équilibre en Thermodynamique, Comptes Rendus de l'Acadèmie des Sciences Paris 108(10), 507-509, 1889.

[32] Gouy, G., Sur l'énergie utilizable, Journal de Physique 8, 501-518, 1889.

[33] Stodola, A., Steam turbine, Van Nostrand, New York, 1905. (translated by L.C. Loewenstein).

[34] Barbero, S., Graziadei, M., Rabuffetti, D., Eventi alluvionali in Piemonte.1316 ottobre 2000. Torino: Agenzia Regionale per la Protezione Ambientale. http://www.arpa.piemonte.it/approfondimenti/temi-ambientali/geologia-edissesto/pubblicazioni/immagini-e-files/evott2000/ 
[35] Wang, Q.A., Maximum path information and the principle of least action for chaotic system, Chaos, Solitons \& Fractals 23, 1253, 2004.

[36] Wang, Q.A., Maximum entropy change and least action principle for nonequilibrium systems, Astrophysics and Space Sciences 305, 273, 2006.

[37] Wang, Q.A., Probability distribution and entropy as a measure of uncertainty, J. Physics A: Math. Theor. 41, 065004, 2008.

Figure 1 - Geographic representation of the area considered

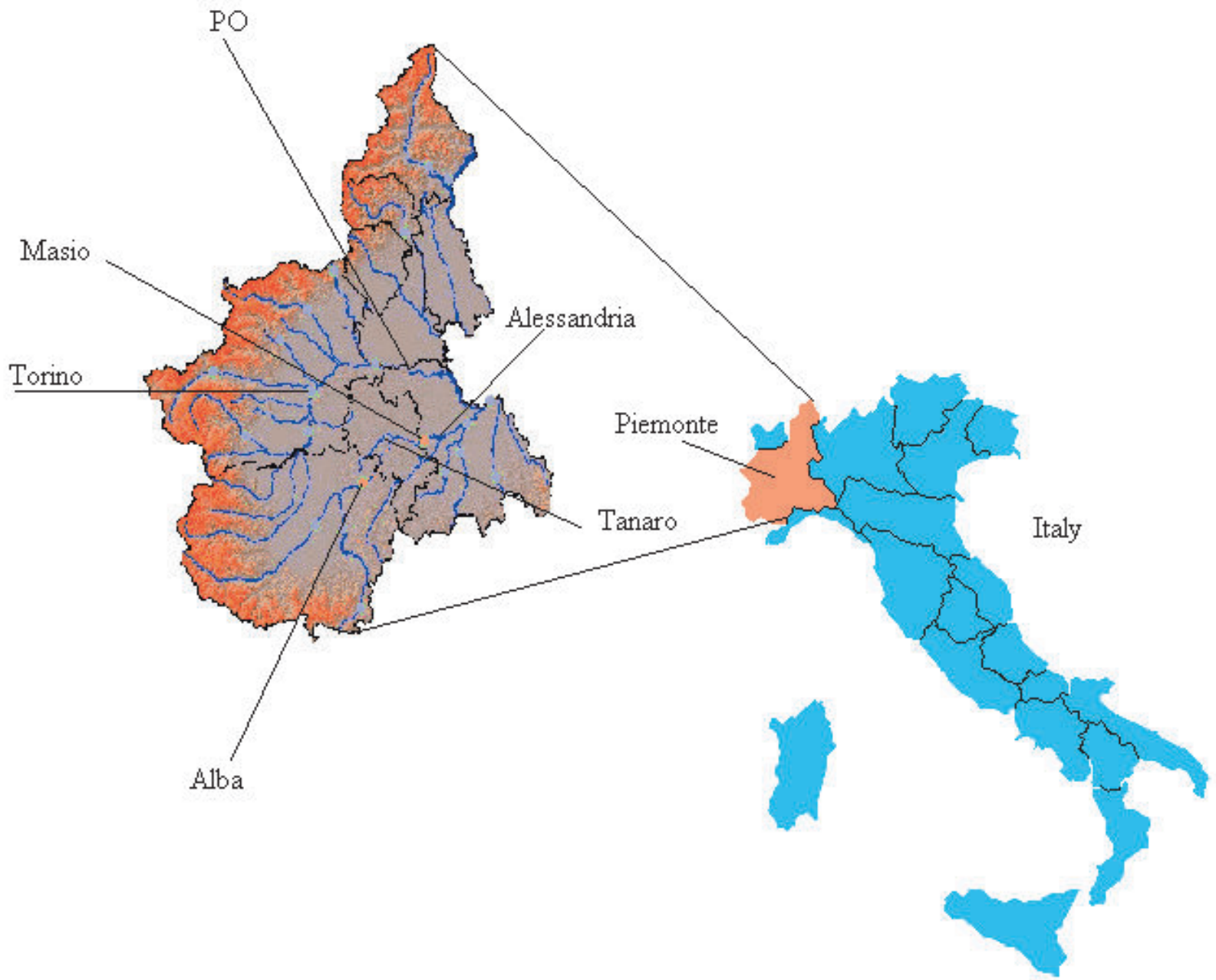


Figure 2 - Entropy generation rate and specific weight in Masio and Alba vs days from the flood inception

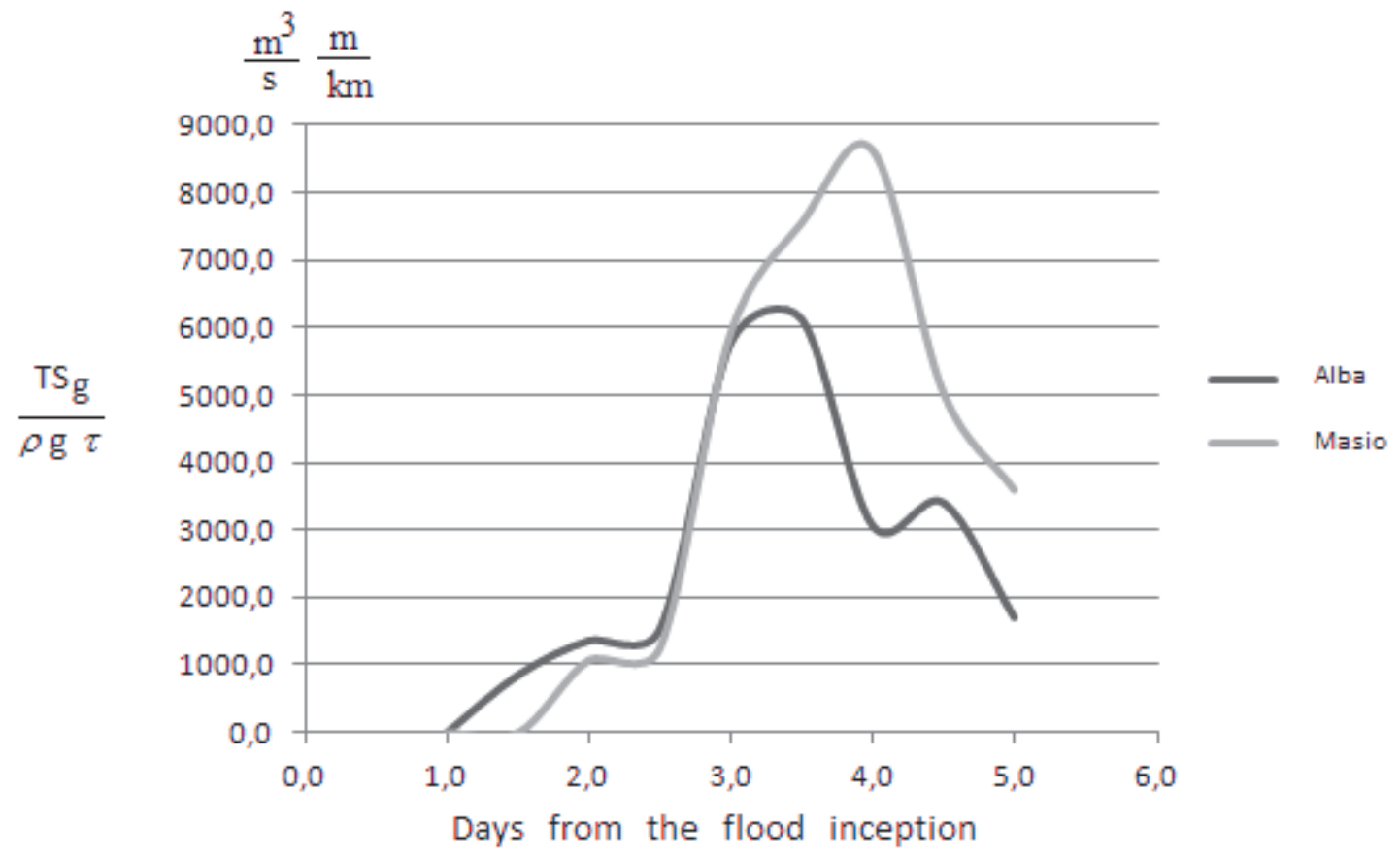

Figure 3 - Specific entropy generation rate variation in Masio and Alba vs days from the flood inception

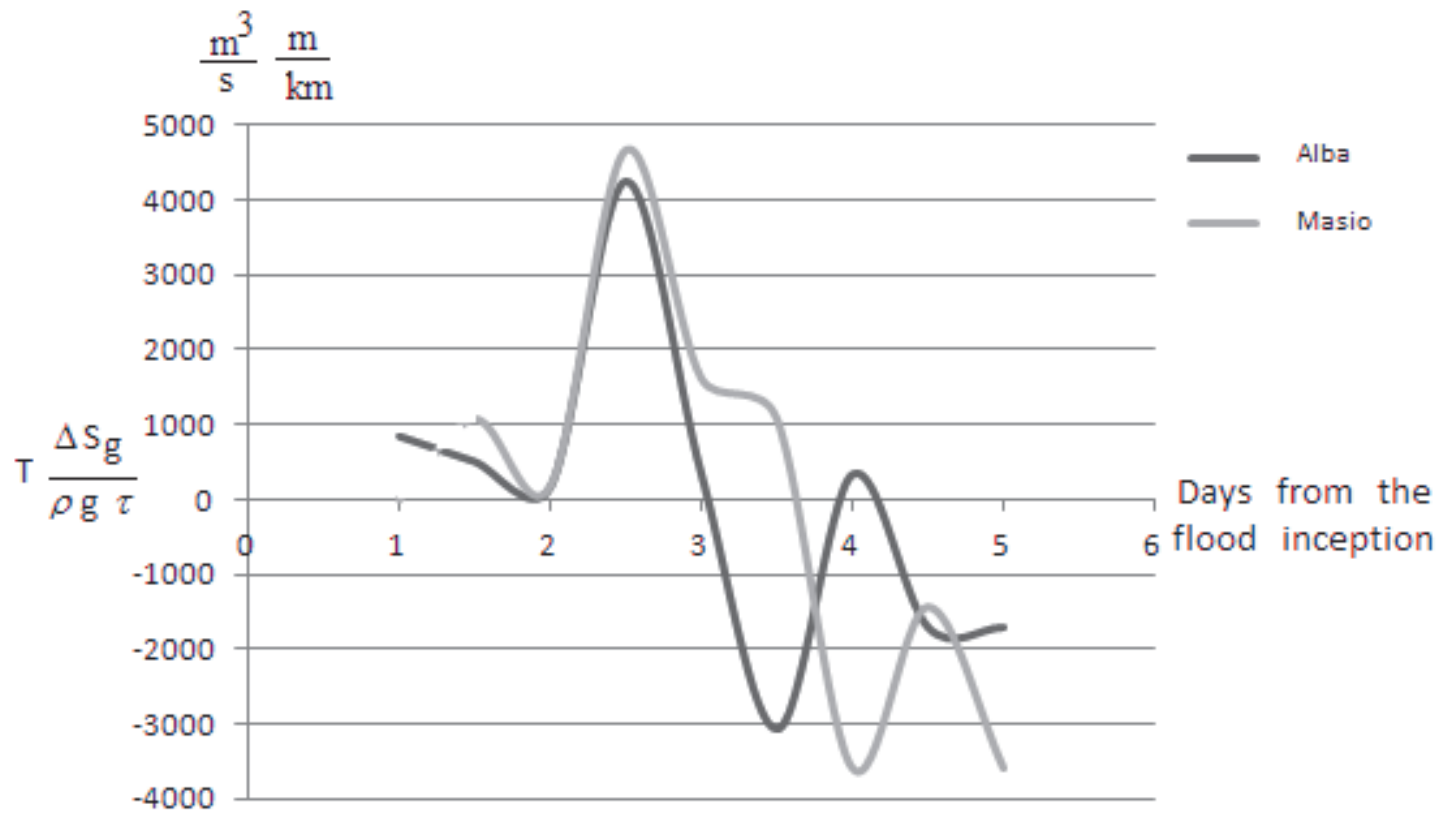


Figure 4 - Entropy generation gradient per unit time and unit mass density and gravity between Masio and Alba vs days from the flood inception

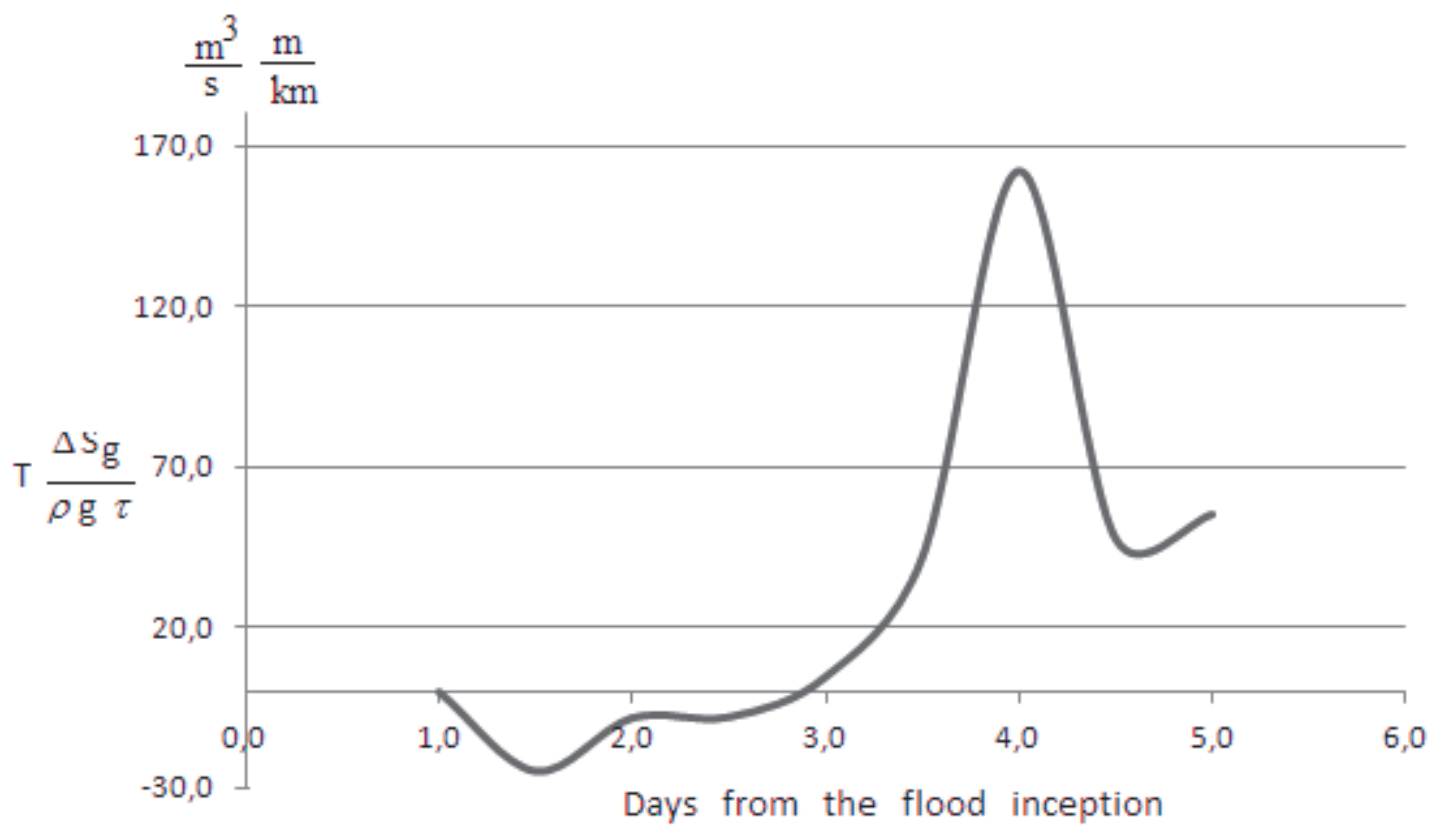

Figure 5 - Flood vs days from the flood inception

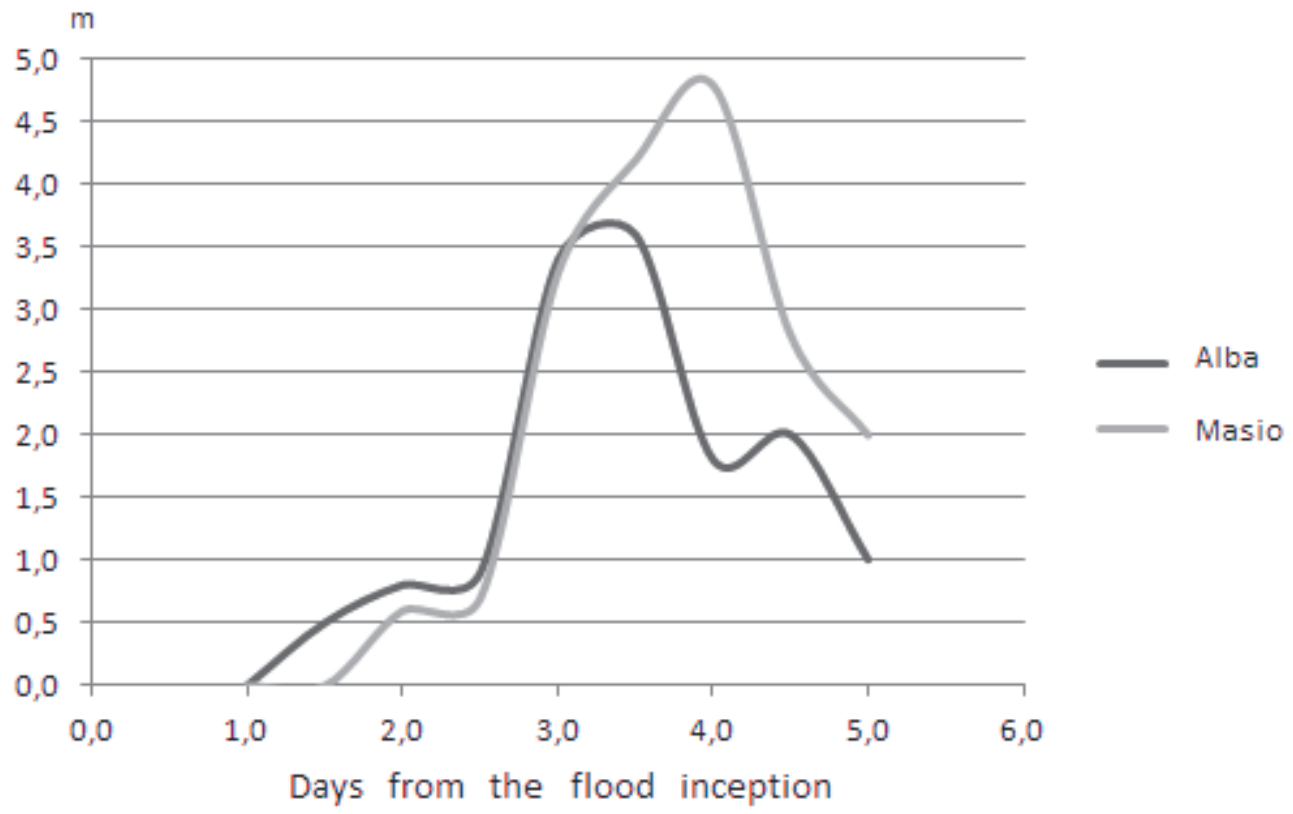


